\title{
Recent Advances in Olfactory Receptor Biosensors and Cell Signaling Cascade Amplification Systems
}

\author{
Dingqiang Lu, ${ }^{1,2,3,4}$ Fuping Lu, ${ }^{1,2}$ Lihua Geng, ${ }^{*}$ and Guangchang Pang $1,2,3,4^{* * *}$ \\ ${ }^{1}$ College of Biotechnology, Tianjin University of Science \& Technology, Tianjin 300457, China \\ ${ }^{2}$ Key Laboratory of Industrial Fermentation Microbiology, Ministry of Education, Tianjin 300457, China \\ ${ }^{3}$ College of Biotechnology \& Food Science, Tianjin University of Commerce, Tianjin 300314, China \\ ${ }^{4}$ Tianjin Key Laboratory of Food Biotechnology, Tianjin 300314, China \\ ${ }^{5}$ Ensoul Technology Ltd. Rm603, Tower B, Lar Valley International, \\ No. 168 Guang An Men Wai Street, XiCheng District, Beijing 100055, China
}

(Received March 24, 2017; accepted October 11, 2017)

Keywords: olfactory receptor biosensors, odorant detection, cell signaling cascade amplification system, GPCRs, biomimetics, electrochemical biosensor

There are a variety of methods to detect odorant molecules, including gas chromatography (GC) and its related coupled techniques, electronic noses, and olfactory receptor (OR)-based biosensors. Nevertheless, studies in which cell and cell signaling amplifier systems were directly utilizied to detect odors are very few. In this review, we aim to provide some references and suggestions for researchers in related fields by summarizing OR sensors and olfactory cell signaling cascade amplifier systems. In this paper, we summarize the detection methods for odorants, introduce the research and progress on OR sensors, and present a proposal of utilizing cell or tissue signaling cascade amplifier systems to amplify electrochemical signals and the use of the $G$ protein signaling cascade amplifier system to prepare electrochemical biosensors. In recent years, the detection technology of electrochemical sensors has matured. Meanwhile, olfactory sensation within organisms basically transfer nerve signals or metabolic endocrine signals in the form of electrochemical signals. Therefore, ORs combined with cell signaling cascade amplifier systems, as well as electrochemical sensors of signal cascade amplifier systems, have incredible prospects for development and application. The proposal of OR electrochemical biosensors and their cell signaling cascade amplifier systems, provides a new idea and a quantitative method for the detection and assessment of odorants and their physiologic processes in the nervous system.

\section{Introduction}

Olfactory sense exists widely in nature. Its significance lies in first, the need to process essential nutrients for survival, second, animals' needs to satisfy their social attributes - for

\footnotetext{
*Corresponding author: e-mail: lhgeng@ensoultech.com

${ }^{* *}$ Corresponding author: e-mail: pgc@tjcu.edu.cn

http://dx.doi.org/10.18494/SAM.2018.1607
} 
example, a single ant tends to blend itself into an ant colony, and third, the need for reproduction

- for example, a male moth can find a pheromone-releasing female from several miles away. ${ }^{(1,2)}$ There are also other needs including avoiding natural enemies; for example, lions and tigers tend to urinate in their own territories to declare their sovereignty. On the basis of these needs, every creature uses its inherent talents to the limit, including not only the sense of smell, but also the senses of hearing, vision, and touch, as well as the magnetic field induction system that birds rely on for their migration. By making use of their own fortes, different species occupy different ecological niches. In this way, a natural law of coexisting harmoniously and adapting to the development of and change in nature is expressed. For determining odorants, through the efforts of scientists, a large number of detection methods have been developed, including gas chromatography (GC), gas chromatography-mass spectrometry (GC-MS), the electronic nose, near-infrared (NIR) spectroscopy and other olfactory receptor (OR)-based biosensors. However, there are very few studies of electrochemical biosensors in which OR cells are utilized as their signal amplifier systems. Burgeoning in recent years, electrochemical biosensors show huge potential in the field of biological molecular detection. This review is a summary of the achievements in the field of determining odorant molecules in recent years and introduces the research progress and practical prospects of OR cell electrochemical biosensors and their cell signal cascade amplifier systems.

\section{GC and GC-MS}

GC, developed in the 1950s, is a segregation analysis method in which gases are used as mobile phases. As it has several advantages, such as high efficiency in segregation, fast analyzing speeds, high sensitivity, and good selectivity, GC has been widely used in many fields and plays an important role in every aspect of modern society. GC consists of the following five systems: a gas path system, a sample entry system, a separation system, a temperature control system, and a detection and recording system, with the separation system and the detection/recording system being its core. With inert gases being used as mobile phases, GC makes use of the fact that the distribution coefficients of components in a sample differ in terms of gas phases and stationary phases. When the sample is brought by a carrier gas into a chromatographic column, the components undergo repetitive alternating distributions between two phases (adsorption-desorption). Components in the stationary phase have different absorbing capacities, so analytes have different speeds when passing through the chromatographic column. After a certain column length, the components in the sample separate from each other and enter the detector. After being amplified, the ion current signals produced by the components will produce a chromatographic peak of each component. In this way, the goal of separate detection is realized. ${ }^{(3-5)}$ Despite having an excellent capability to detect odorants, GC is not an olfactory sensor. In addition, owing to its high price and rather high demand for skilled experimental operators, GC is mainly used in assessments of complicated mixtures. In such a background, the electronic nose technology for odorant detection emerged in a timely manner. 


\section{Electronic Noses}

The unceasing progress of science and technology has made human beings no longer satisfied with judging odorant characteristics of things through their own sense of smella rather imprecise method. With the constant deepening of our understanding of the process of smelling and the continuous development of sensor technology, the time was ripe for the emergence of electronic nose technology. In the early 1980s, Zaromb and Stetter ${ }^{(6)}$ took the lead in exploring array theory of sensors and applied arrays to the detection of inflammable and poisonous gases. Meanwhile, Persaud and Dodd, ${ }^{(7)}$ who applied arrays to the analysis of multiple organic volatile gases, opened the door to the study of electronic noses. Electronic nose technology is the most commonly used method at present for odorant detection, and is applicable to gas-sensitive systems for measuring one or many odorants. ${ }^{(8)}$ Compared with traditional technologies for analyzing odors, such as GC, mass spectrometry (MS), and flame ionization detection (FID), the electronic nose has more advantages; it is fast, convenient, and economical, hence it is widely used in fields such as food, medicine, agriculture, environment monitoring, and public safety. A typical electronic nose consists mainly of three parts: ${ }^{(9)}$ a headspace sampler, which introduces the gas from the headspace in a sealed bottle containing a sample to the host, which contains a sensor array; a gas sensor array, which generates an instant response to a gas that has an odor in the analytical sample, with the response intensity gradually becoming stronger and then weaker until it comes to a stable status; a signal processing system, also known as a pattern recognition system, which preprocess the information acquired by the gas sensor array, extracts the features of the information, and carries out a variety of statistical analyses using software. The sensor array and the pattern recognition system are the core elements that determine the working efficiency of an electronic nose.

According to different working principles, sensors can be classified as piezoelectric sensors, electrochemical sensors, optical sensors, and thermal sensors, for example. Taking the metal oxide sensor (MOS) as an example, metal oxides such as $\mathrm{SnO}_{2}, \mathrm{ZnO}, \mathrm{Fe}_{2} \mathrm{O}_{3}$, and $\mathrm{WO}_{3}$ belong to n-type semiconductor materials because they have a large number of electron holes on their sensitive membrane surfaces, that can be restored easily by gases such as $\mathrm{H}_{2}, \mathrm{CO}, \mathrm{CH}_{4}$, and $\mathrm{H}_{2} \mathrm{~S}$ at temperatures from 300 to $500{ }^{\circ} \mathrm{C}$. When the sensitive membrane acquires electrons, conductivity changes. For a particular gas, the variation in its electrical conductivity is related to its concentration. To reduce the working temperature of metal oxide gas sensors and to increase their sensitivity, precious metals such as platinum, gold, and palladium are often mixed into metal oxide sensitive materials during their preparation. At present, the technique for manufacturing MOS sensors has been perfected, resulting in successful commercialization. MOS sensors have a high reproductivity in terms of their manufacture and high sensitivity.

Electronic noses are widely used in fields such as food analysis, medicine, and environmental detection. Supriyadi et al. found that electronic noses could detect and distinguish the different maturation periods of salak. ${ }^{(10)}$ They also used a GC-odor detector to determine ten characteristic components that could influence odors and analyzed maturity-related changes in components by MS online analytical processing (OLAP) with the use of an electronic nose. Italian researchers Buratti et al. ${ }^{(11)}$ provided sensory descriptions of wines of different Italian 
brands by combining an electronic nose with a spectrophotometer. They applied a genetic algorithm to choose variables and established a forecasting regression model. For the selected model, they adopted precise validation technology to test the output steps. Clinically, electronic noses are used to evaluate halitosis. ${ }^{(12)}$ They provide useful analytical controls by analyzing the breath $^{(13)}$ exhaled by patients with diabetes, as well as analyzing their urine. ${ }^{(14)}$ They have an accuracy as high as $88 \%$ in detecting infections of the ear, nose, and throat. ${ }^{(15)}$ They are also used to evaluate unpleasant smells on tablets, ${ }^{(16)}$ the renal functions of patients, ${ }^{(17)}$ and the body odor of mental patients. ${ }^{(18)}$ Another application of the electronic nose in medicine is to detect cancer. Italian researchers Bernabei et al. proposed a noninvasive diagnosis of early urethral carcinoma using electronic noses. ${ }^{(19)}$ D'Amico et al. ${ }^{(20)}$ pointed out that electronic noses could distinguish benign lesions from melanoma or black cancer; Machado et al. ${ }^{(21)}$ successfully diagnosed lung cancer by applying the Cyranose-320 electronic nose. Compared to GC, electronic noses not only need no complicated pretreatment processes for samples, but also have several advantages such as requiring small volume, having low cost, and being highly reliable. However, recognition by electronic noses of odors is still merely the recognition of compounds, making it different from real olfactory sensation.

\section{NIR Spectrum Technology}

The NIR spectrum is the electromagnetic spectrum between the visible-light (VIS) region and the mid-infrared (MIR) region. As defined by the American Society for Testing Materials (ASTM), NIR is the region between 780 and $2526 \mathrm{~nm}$. It was the first invisible region discovered in the absorption spectrum. The NIR technique is a method in which chemical bonds $(X-\mathrm{H})$ that contain hydrogen groups $(X-\mathrm{H}$, with $X$ being $\mathrm{C}, \mathrm{O}, \mathrm{N}$, or $\mathrm{S})$ are utilized to stretch out and relax frequencies, resulting in vibration frequencies and combined frequencies. A stoichiometric multivariate calibration method has been adapted for the analysis of absorption spectra in the NIR region, that enables the NIR absorption spectrum of the calibrated sample to be associated with its composition in order to establish a correlation-calibration model to show the correlation between the calibrated sample's absorption spectrum and its concentration or its structure. When making a prediction about an unknown sample, the concentration or the properties of the sample can be quantitatively predicted by applying an established calibration model to the absorption spectrum of the sample. ${ }^{(22,23)}$ Fourier transform infrared spectroscopy (FTIR) requires a light splitting device and a means of scanning for the analysis of gases. FTIR can measure and analyze the concentrations of toxic gases over a wide infrared region. On one hand, this method has high sensitivity and can realize simultaneous analysis and measurement of a variety of gases. On the other hand, it requires gas sampling methods, and the measurement and analysis of gases can only be carried out in a laboratory, so it is impossible to use FTIR to realize online real-time detection of the gases, for example, on the scene when a fire breaks out. In recent years, with the development of semiconductor laser devices and the expansion of research on laser spectroscopy, research on measurement methods based on laser absorption spectra of NIR semiconductors has attracted great attention in various countries and fields. ${ }^{(24-26)}$ 


\section{Biomimetic Olfactory Sensors}

The biological olfactory system can recognize and discriminate thousands of volatile organic compounds (VOCs) with extremely high sensitivity and specificity. The most fundamental elements are ORs in the cilia of olfactory sensory neurons (OSNs), and they contribute greatly to the high-performance olfactory system. The excellent properties of ORs are generally recognized in the development of biomimetic OR-based biosensors. Over the past two decades, much work has been done in developing OR-based biosensors because of their promising potential in many applications. In this section, we will outline the latest advances of some ORbased biosensors. $^{(27)}$

\subsection{Microcantilever biosensor}

Microcantilever biosensors (Fig. 1) are a new technology developed from atomic force microscopes and biosensors. Compared with traditional sensors, microcantilever biosensors have several advantages including high sensitivity, fast response speed, good reproducibility, label-free analysis, and real-time monitoring, so they have quickly become a topic of considerable research interest in the sensors field and thus hold promise for practical applications. As shown in Fig. 1 (slightly modified from literature). ${ }^{(29,30)}$ The sizes of microcantilevers vary from the millimeter to the nanometer scale. The smaller the size, the higher the sensitivity of the microcantilever. A micro- to nanoscale size sensor can detect mass changes from pg to fg, which means a large enhancement compared with the limits of detection of other kinds of sensors. Kosaka et al. ${ }^{(31)}$ realized the detection of cancer markers in serum using a dynamic mode and achieved a limit of detection of $1 \times 10^{-16} \mathrm{~g} / \mathrm{mL}$. However,

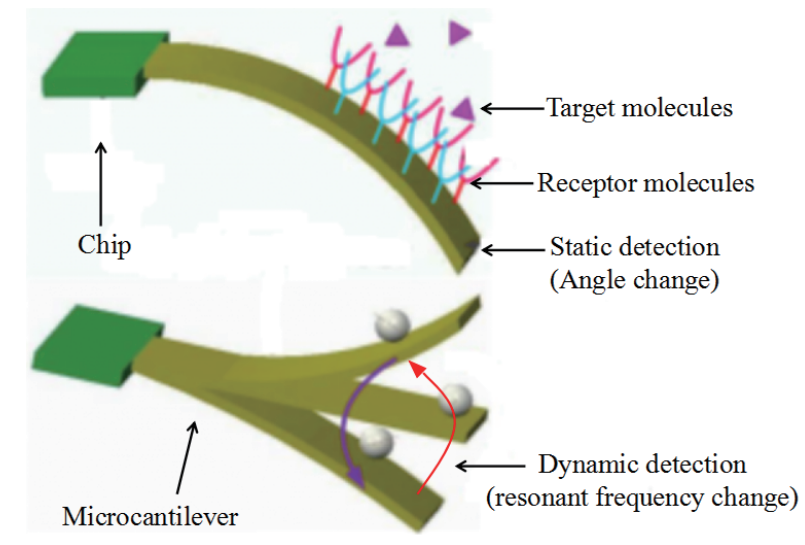

Fig. 1 (Color online) Microcantilever biosensor operating modes and principles. Immobilize one end of a microcantilever and set the other end free. After trimming, the surface on one side of the free end absorbs to-bedetected molecules, causing a difference between the stresses on the upper surface and the lower surface and thus leading to a bending deflection of the free end or a change in the oscillation frequency. This change is related to the mass of the adsorbed molecules to some extent. The optical lever method, piezoelectric method, piezoresistive method, and capacitance method for example, are often applied to the measurement of such changes. ${ }^{(28-30)}$ 
owing to the influence of a damping phenomena, it is difficult for a microcantilever biosensor with a micro to nanoscale size to function stably in a liquid environment. For this reason, microcantilever biosensors are more typically applied to the detection of gas molecules in air. Their microstructure has given microcantilever sensors other advantages, such as small volume and low cost, easy assembly, and portability. As for microcantilevers, the design of their shapes and sizes, the choice of construction materials, the thickness of their surface sensitive layers, and the trimming of their sensitive membranes can directly impact the performance of sensors in detection. In terms of detecting one-component substances, many reports on research on microcantilever biosensors have appeared. ${ }^{(32-34)}$ However, at present, the improvement of the sensitivity of self-controled microcantilever sensors and shortening their response time are still the main tasks in the future faced by scientists.

\subsection{Electroantennography (EAG) techniques for insects}

At present, EAG is an electrophysiological method widely used to directly detect insect responses to chemical signals of volatile substances. The olfactory function of insect antennae plays a crucial role in positioning their hosts, foraging, searching for mates, and choosing proper sites to lay eggs. ${ }^{(35-37)}$ Schneider ${ }^{(38)}$ was the first to apply EAG to detect antenna responses for sex pheromones and acquire the cumulative receptor potential of slow gradual changes (namely, EAG). EAG screens out compounds to which insects have physiological responses from a series of chemicals. As EAG has greatly reduced the workload, it plays a crucial role in exploring insect sex pheromones and insect responses to plant volatiles and their perception of plant odors.

Although similar to an EAG response recording device, a single-cell-response recording device is slightly different in the design of its amplifier. Single-cell responses include AC nerve impulse responses and DC receptor potentials, whose passbands are different. The passband of the former is about 40 to $500 \mathrm{~Hz}$ and that of the latter is about 0 to $40 \mathrm{~Hz}$. Røstelien et al ${ }^{(39)}$ used the antenna neuron receptor cells of Myzus persicae (Sulzers) to detect their responses to germacrene D. As for the trichoid sensillum, which is sensitive to sex pheromones, every trichoid sensillum usually has several kinds of OR cells, and each kind of OR cell is only sensitive to one kind of odorous compound, so its sensitivity is high; an amount of an odorous stimulus as low as $10^{-4}-10^{-3} \mu \mathrm{g}$ can cause an obvious response. Insect antenna cell receptors have very strong selectivity and specificity for the components of sex pheromones, as well as an uncommon structure-activity correlation.

The combined technique of electroantennography-single sensillum recording (EAG-SSR) has been widely used in studies of insect antenna peripheral nervous systems for feeling, processing and coding the masses, and space-time distributions of volatile odors. ${ }^{(40)}$ With the help of technologies such as electrophysiology, Bau et al. ${ }^{(41)}$ studied EAG responses with respect to changes in the impulse frequencies of sex pheromones, the temperature, and the humidity, and they also studied the specificity of insect olfactory responses to the impulse frequencies of sex pheromones. Vickers et al. also conducted research on related aspects. He fixed an antenna of an insect to the head of another insect. Attached with an electrode, the antenna recorded the electrophysiologic responses of another insect's antenna to sex pheromones when it was flying against the wind in a wind tunnel. Afterwards, he performed SSR to detect whether the insect was influenced by the space distribution of sex pheromones when it flew in the wind tunnel. ${ }^{(42)}$ 
Apart from gas chromatography-single sensillum recording (GC-SSR), GC-EAG has developed rather rapidly in recent years. One of EAG's prospects is that it can create practical value for products. For example, it can provide data guidance for the synthesis of insect attractants, sex lures, and repellents. At present, EAG has an obvious defect-because it is limited by its stimulative gas control system, its receptor cannot determine the concentrations of odor molecules that have reached it. This defect has seriously impaired the accuracy of experiments. ${ }^{(43)}$ In addition, electrophysiological experiments cannot simulate the intensity of the insect response to odors under natural conditions.

\subsection{Biomimetic olfactory sensors based on quartz crystal devices}

A quartz crystal microbalance (QCM) is a device for determining the change in pressure on a crystal surface through the measurement of the change in the resonance frequency of the surface on the basis of the principle that a subtle change in pressure on a piezoelectric crystal surface leads to a change in the resonance frequency. Because of this principle, a QCM can be used to determine the change in the mass of a substance adhered to the surface. Meanwhile, a QCM has several advantages including high sensitivity, fast response speed, and the capability of real-time dynamic detection. Therefore, QCMs have been widely used for detecting interactions between odorant molecules and ORs, as shown in Fig. 2 (slightly modified in accordance with information in the literature). ${ }^{(44)}$

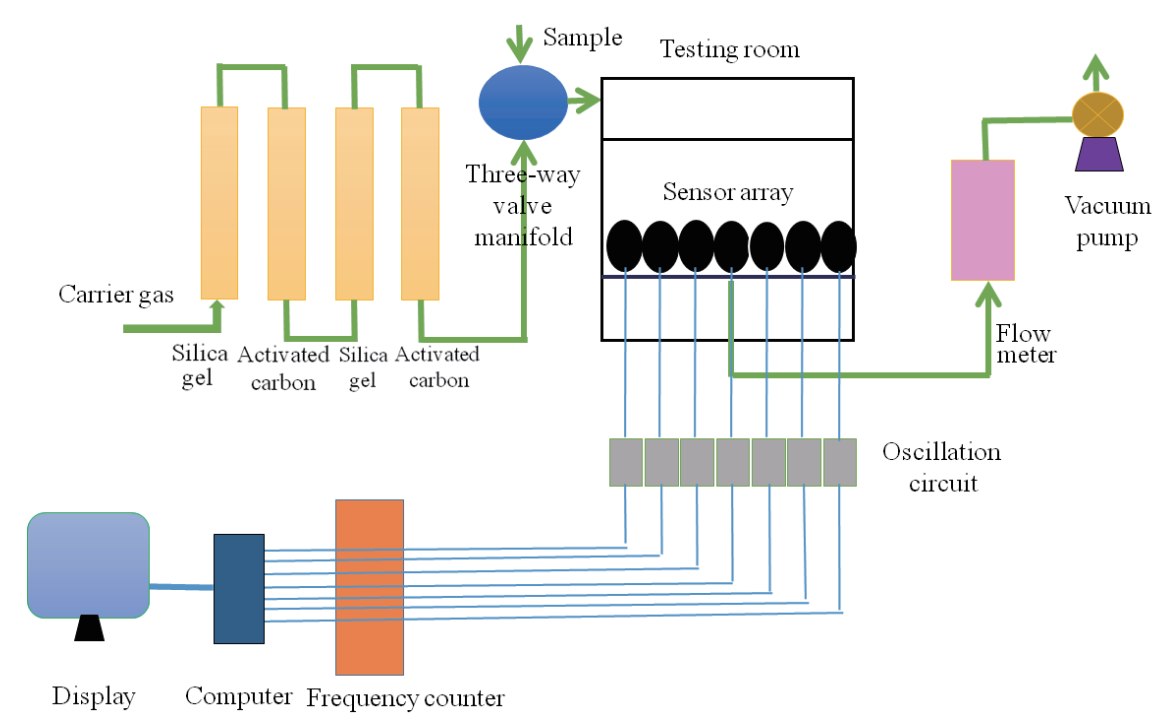

Fig. 2 (Color online) Olfactory sensor based on QCM. When particular molecules (such as gases) are absorbed by a substance (such as an OR) and fixed on its surface, the change in the mass causes a change in the resonance frequency of the quartz crystal, and the change in the frequency will be proportional to the change in the mass, namely, $\Delta F=K \Delta m$. In this formula, $\Delta F(\mathrm{~Hz})$ is the change in the QCM frequency, $\Delta m(\mathrm{~g})$ is the change in the mass of the absorptive substance on the surface, $K$ is the correlation coefficient. $K=-F /(A r t)$, where $F(\mathrm{MHz})$ is the original frequency of the crystal, $A\left(\mathrm{~cm}^{2}\right)$ is the overall surface area of the electrode, $r\left(\mathrm{~g} / \mathrm{cm}^{3}\right)$ is the density of the crystal, and $t(\mathrm{~cm})$ is the thickness of the crystal. After a film has formed on the electrode surface of the QCM, the two changes are still subject to the correlation of $\Delta F \propto \Delta m$, and the correlation coefficient $K$ is not only decided by the nature of the quartz crystal but also correlated to the film's characteristics. ${ }^{(45)}$ A considerable amount of literature has been published on this aspect. ${ }^{(44,46)}$ 


\subsection{Biomimetic olfactory sensors based on field effect transistors (FETs)}

An FET is a commonly used semiconductor amplifier, where the basic principle of operation is to control the movement of most of the carriers in the device by utilizing the electric field effect of the semiconductor's surface or interior. Fromherz et al. ${ }^{(47)}$ coupled single neurons with a bare FET (without a metal grid), used a stereo microscope and a glass micropipette to absorb cells into the transistor, and inserted the glass microelectrode into the cells to stimulate the cells and record their intracellular voltage. That study was the earliest one in which an FET was applied to a cell sensor. Schütz et al. ${ }^{(48)}$ built a biomimetic olfactory sensor (Fig. 3 slightly modified in accordance with information in the literature) by coupling the antennae of Colorado potato beetles to FETs through a biological electronic interface. The sensor was used to detect the damage caused to the plants inside a greenhouse under real conditions. As shown in the findings, the biomimetic olfactory sensor had a rapid response with a rather large dynamic range and a low detection limit to cis-3-hexen-1-ol, the volatile gas emitted from the plants after they had been damaged. This system can reveal whether the damage to a greenhouse, where 1000 plants are growing, is caused by a single mechanical force or by beetles. This kind of biomimetic olfactory sensor can be used to protect plants by indicating invading pathogens and can also be used as a quality control tool to protect food in storage.

\subsection{Biomimetic olfactory sensors based on microelectrodes}

Insect antennae contain olfactory neurons. When an antenna is exposed to gas, the stimulation of odorant molecules is first received by olfactory neurons and then is transduced into a string of action potentials travelling along the olfactory neuron axon. The frequencies of the action potentials produced by the olfactory neurons before and after they receive stimulation are different: From the resting state before receiving stimulation to the state of excitement after receiving stimulation, the frequency of the action potentials rises from about $20 \mathrm{pps}$ to about

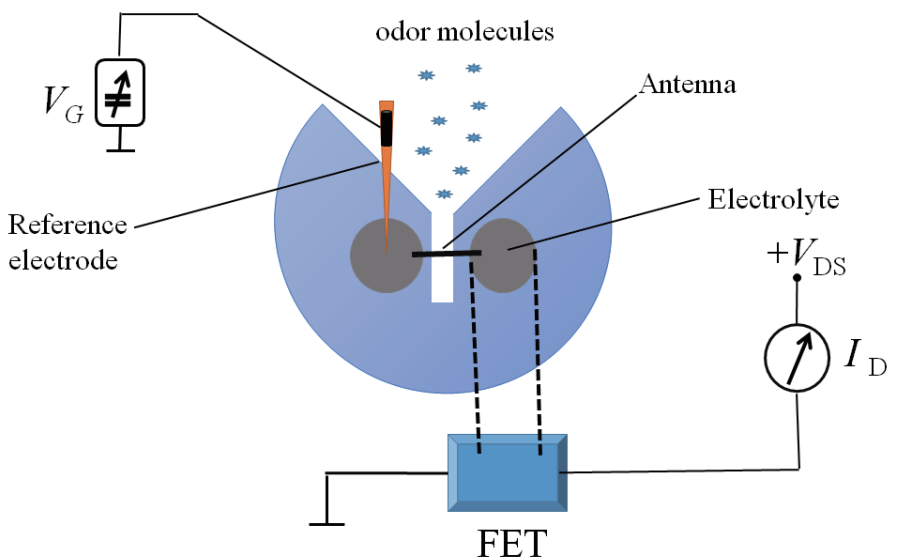

Fig. 3 (Color online) Olfactory sensor based on FETs. 
$300 \mathrm{pps}$, and the frequency is related to the gas concentration. The measured frequency of action potentials reflects the intensity of gas stimulation. Huotari ${ }^{(49)}$ used microelectrodes to record the frequencies of the action potential of Calliphora olfactory receptor neurons (ORNs) in the resting state and in the excited state (Fig. 4) to estimate the concentrations of the gases acting on the antenna ORNs. As shown in the findings, Calliphora ORNs have rather high sensitivity and specificity to three odorant molecules, which are 1,4-diaminobutane (1,4-DAB), 1-hexanol (HX), and butanoic acid (BA), but have no sensitivity to other tested odorants. The minimum concentrations of gases that can transform the action potential of ORNs from the resting state to the excited state that can be distinguished by the system are $1 \mathrm{~nL} / \mathrm{L}$ for $1,4-$ $\mathrm{DAB}, 8 \mu \mathrm{L} / \mathrm{L}$ for $\mathrm{HX}$, and $20 \mu \mathrm{L} / \mathrm{L}$ for BA. When the concentration of a gas is higher than a certain amount, the olfactory neurons stop producing an action potential, which perhaps results from the adaptation and saturation of the olfactory neurons. As shown in the findings, this kind of sensor is suitable for the detection of odors and can be used to study the characteristics of olfactory neuron response to gas stimulation. Shimizu and Stopfer studied the connections among different ORNs exposed to outside stimulation. The results showed that spiking in one ORN can inhibit or activate the responses of other ORNs in the same sensillum. Synergy exists among neurons, which means a single-cell-based microelectrode sensor is unable to show the real action between cells and ligands. ${ }^{(50)}$

\subsection{Biomimetic olfactory sensors based on light addressable potentiometric sensors (LAPSs)}

LAPSs commonly comprise semiconductor chips. As established in many studies, LAPSs can be used to build cell-semiconductor compound systems for monitoring extracellular electric potentials. ${ }^{(51,52)} \mathrm{Xu}$ et al. ${ }^{(53)}$ used LAPSs to monitor single excitable cell membrane electric potentials without damage. LAPS technology has many advantages. For example, LAPS surfaces are so smooth that they can measure flowing fluids easily, LAPS manufacture is very

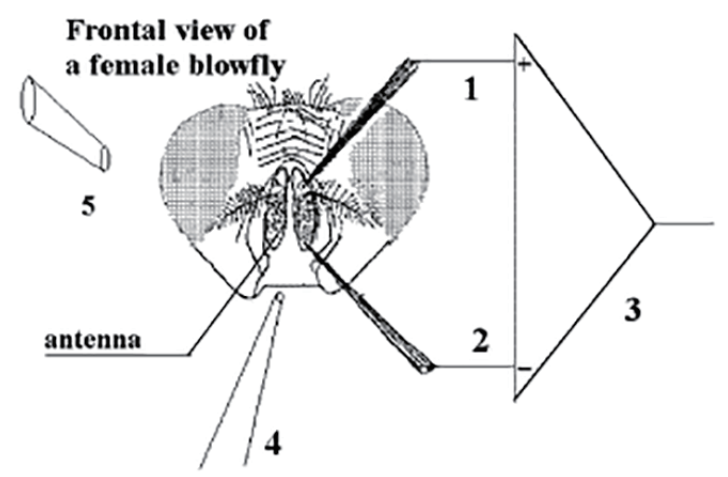

(a)

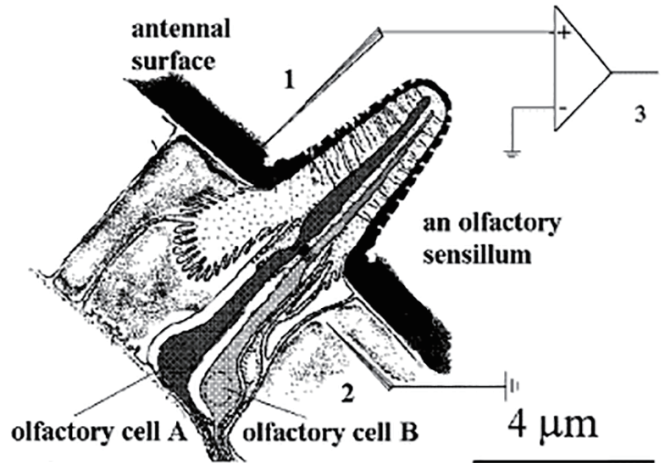

(b)

Fig. 4 Olfactory sensor based on microelectrode. (a) Head of a female blowfly (frontal view) with measurement microelectrode 1 and reference microelectrode 2 on the surface of the right antenna, amplifier 3, exposure airflow tube 4, and clean airflow tube 5. (b) Olfactory sensillum and its two sensor cells (A and B), and electrodes as in (a). ${ }^{(49)}$ 
simple, LAPSs have a long service life, and LAPSs can be used for addressable measurement at any location of the chip surface. LAPSs are detectors that utilize the photoconductivity of semiconductors to measure voltages on surfaces. ${ }^{(54)}$ When an appropriate amount of light irradiates LAPSs, electron-hole pairs emerge owing to the intrinsic absorption of the semiconductor. When the semiconductor surface in contact with an insulation layer is in a state of depletion, under the action of the strong electric field in the depletion region, the photoinduced electrons and the electron holes, which had entered the depletion region through diffusion, are separated and then gather on both sides of the depletion region. In this way, a photovoltage is generated. If the light is a laser beam modulated to a constant state, the photovoltage is alternating. Afterwards, because of the insulating layer capacitance, an alternating current passes across the external circuit. When the properties of the light (wavelength and power), the resistivity of the silicon substrate, the thickness of the silicon wafer, and the size of the sensor chip are within certain ranges, the size of the photovoltage depends on the thickness of the depletion layer. The thicker the depletion region, the more photon-generated carriers it can collect, and the higher the photovoltage. Ideally (regardless of the state of the interface and the electric charge on the oxide layer), the thickness of the depletion layer is determined only by the equivalent offset. When the externally impressed bias voltage is constant, the thickness of the depletion layer is determined only by the extracellular potential that has been coupled on the depletion layer (Fig. 5). Therefore, the change in the extracellular potential can be recorded by focusing light spots on the target cells on the LAPS surface and measuring the local surface potential of the illuminated region.

Apart from these, there are olfactory sensors utilizing technologies such as surface plasmon resonance (SPR), fluorescence [including fluorescence resonance energy transfer (FRET)], luminescence, bioluminescence [including bioluminescence resonance energy transfer (BRET)], chemiluminescence, surface acoustic waves (SAWs) and resonators, on which we will not go into detail. A comparison of the several kinds of sensors mentioned above confirms that olfactory sensors, which utilize QCMs or SPR technology, work under the principle that, after

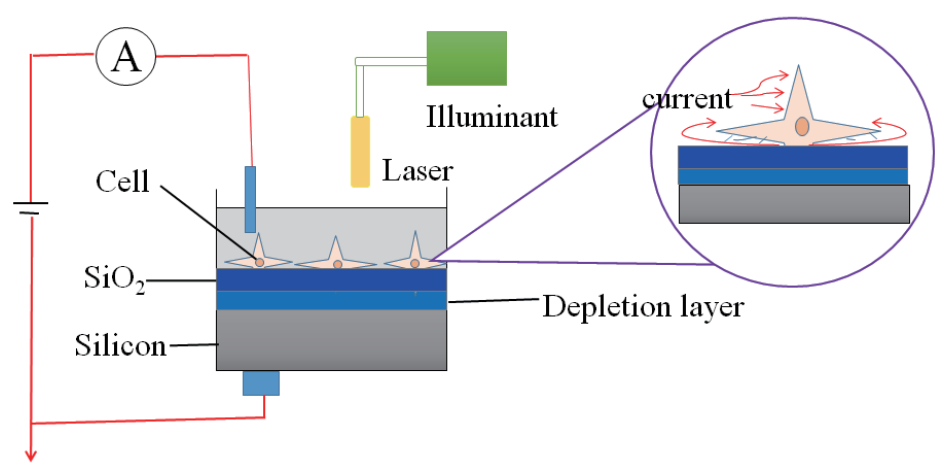

Fig. 5 (Color online) Olfactory sensor based on LAPSs and the principle of cell biological sensors that utilize LAPS technology. 
ORs combine with corresponding odorant molecules, their mass or optical properties change. Whether the odorant molecules have combined with ORs and how many have combined can be determined by measuring the extent of the change. As for ligand-receptor interaction in a "one-to-one" mode, these two technologies have already met the requirements. However, the OR-odor combination is a "many-to-many" mode, and the information lies more in the neural electric signals that form after ORs have combined with an odor; this information cannot be acquired using these two kinds of sensors. As for FET olfactory sensors, when multiple ORs are fixed to a grid electrode, the change in the electric charge on the grid electrode surface is the superposition of the electrodischarging effects of all the ORs, so the drain current reflects the total electrodischarging of all ORs, with no information about the electrodischarging of a single OR. Microelectrode olfactory sensors also have unique defects. First, it is difficult for a microelectrode to record the electrical activities of many ORs simultaneously. Usually, odorant information is coded by a large number of ORs simultaneously. However, under a microscope, four micropositioning microelectrodes at most can be juxtaposed at the same time, which means data at four locations can be recorded simultaneously. Second, it is not easy for a microelectrode olfactory sensor to record for a long time. Because of ground vibrations and airflow, the recording tip drifts slowly, which leads to inaccuracy in the location if the sensor has recorded information over a long period. A multielectrode array sensor is an ideal tool, as it can collect electrical activities of dozens or even hundreds of ORs simultaneously, providing a large amount of data for studying neural coding. As the cells being detected are attached to the surface of the electrode coating, there is no problem of a drifting tip. Thus, it is easy for a multielectrode array sensor to record over a long time. However, as cells do not achieve a oneto-one correspondence with electrodes, the data on an electrode may be the superposition of electrical signals of multiple ORs, which causes difficulties in data analysis in the next step.

\section{Electrochemical Biosensor}

In an electrochemical biosensor, biological molecules or organisms themselves are used as the molecular recognition element, an electrode as a signal converter, and resistance or current as the characteristic detection signal. For an organism itself, signal transduction in its activity is neither an optical nor a sound or vibration wave; it is, after all, electrochemical. Consquently, the electrochemical biosensor is the closest to the existing natural signal transferring system in life. Taking the cell as an example, as the smallest unit of life activity, after the effect of external substances, some of ligand molecules are combined with the receptors on the surface of cell membranes, which leads to a change in the receptor conformation, and further, to changes in the chemical substance composition and ion concentration in the cell via the activation of the transmission paths of several kinds of signals in the cell. Another signal transmitting pathway is to transport substances into cells via the transport proteins or ion channels that normally provide the materials and energy for cell metabolism. The communication between cells also depends on electrochemical actions, such as the transduction between neurotransmitters and electrical signals during the transduction of signals mediated by neuronals. In short, the various 
processes of life are strongly dependent on electrochemical reactions. Using electrochemical methods to study the interactions between biomolecules has more advantages and broader application prospects than any other method.

Compared with other sensors, an electrochemical biosensor can directly transform the interactions between biomolecules into electric signals. Its advantages include simple instrumentation, low price, easy operation, quick response, and the capability of directly acquiring complicated systematic information, all of which make the electrochemical biosensors a major focus of research in the field of biosensors. In recent years, there has been an increasing amount of literature in this area. ${ }^{(55-58)}$

\section{OR Cell Electrochemical Biosensor}

Devoted to research on biosensors over the years, our laboratory has made a breakthrough in research on nanometer sensors. Kang et al. ${ }^{(59)}$ used thionine-chitosan, the eletronic medium of cross-linking horseradish peroxidase (HRP), as the cross-linking agent to absorb nanogold into a glassy carbon electrode and prepared an $\mathrm{H}_{2} \mathrm{O}_{2}$ biosensor based on the absorption and fixation of nanogold to HRP. The results showed that the linear range of the biosensor detection was $1 \times 10^{-7}-1 \times 10^{-4} \mathrm{~mol} / \mathrm{L}$, and the limit of detection was $5.0 \times 10^{-8} \mathrm{~mol} / \mathrm{L}$. The biosensor was excellent in terms of service life, stability, reproducibility, and selectivity. On the basis of this, using chitosan as the cross-linking agent, we adopted nanogold-thionine to absorb the HRP electrochemical signal amplifier system and used Bacillus cereus monoclonal antibodies as the biological molecular recognition element. Thus, two-layer nanogold-trimmed Bacillus cereus electrochemical immunosensors were built. The results indicated that the sensor's response current and bacterial concentration presented a linear correlation within the range of $5 \times 10^{1}$ to $5 \times 10^{4} \mathrm{cfu} / \mathrm{mL}$, with a correlation coefficient of 0.9966 and a limit of detection of $10 \mathrm{cfu} / \mathrm{mL} .^{(60,61)}$ This has laid a foundation in the realization of the rapid quantification and online detection of Bacillus cereus.

The significance of this technology lies in the fabrication of a nano-immunosensor for combination with Balb/c mouse monoclonal antibodies as well as an HRP signal amplifier system. As a matter of fact, antibodies are immunoglobulin. The interaction between immunoglobulin and antigen is basically the same as that between a receptor and a ligand. It is reasonable for us to speculate: now that we can transform the weak interaction between antibody and antigen to an electrochemical signal, we could also transform the signals between other receptors and ligands to electrochemical signals so that we can develop electrochemical receptor sensors. Our team applied this signal amplifier system to studies of electrochemical gustatory receptor sensors and made some breakthroughs. On one hand, we applied this amplifier system to studies on mouse GPR70 (T1R1, NCBI Code: AF337040.1) umami electrochemical biosensors, which means we carried out gene synthesis by the oligo method, connected GPR70 gene to the eukaryotic expression vector pcDNA3.1 through proteolytic cleavage, transformed the bacterial strain DH5 $\alpha$ to acquire a plasmid vector, used lipidosome lipofection to transform it to a CHO-K1 cell, cultivated it and made it express the target protein GPR70. Afterwards, we utilized multiple thiols in the structural domain within the 
receptor cells to connect nanogold-thionine-chitosan, adopted HRP to further amplify the signals showing the interaction between the extracellular structural domain and the ligands, and developed a mouse GPR70 electrochemical umami sensor. As demonstrated in practical testing, this umami receptor sensor can detect sodium glutamate and a variety of tricarboxylic acid (TCA) metabolic intermediate compounds $\left(5 \times 10^{-14}-7 \times 10^{-12} \mathrm{~mol} / \mathrm{L}\right)$ and presents a hyperbolic kinetic equation similar to an enzyme-substrate one. Then we carried out regression based on the hyperbola with a correlation coefficient of 0.9745 . When the concentration of sodium glutamate was $5 \times 10^{-14}-1 \times 10^{-12} \mathrm{~mol} / \mathrm{L}$, the difference between the concentration of sodium glutamate and the detected signal showed a linear relationship with a correlation coefficient of 0.9915 ; by conducting a regression analysis on the experimental results by the double reciprocal method, the concentration/activity constant (the ligand concentration at half the maximum activity) of the receptor-ligand interaction was $1.3164 \times 10^{-12} \mathrm{~mol} / \mathrm{L}$ (unpublished data). The advantage of this method lies in using immobilized nanogold-HRP to replace the intracellular signaling pathways and signal amplification system, thus avoiding "crosstalk" between the complex intracellular signaling pathways. By this method, it is easy to realize high flux, but there is one obvious disadvantage: the method fails to reflect the interaction among the receptors on the cell membrane because of fluidity and real biological effects generated from the "crosstalk" transmitted by cell signals and among a number of signaling pathways in cells.

To detect the real biological effects generated by the interaction between receptors and ligands more accurately, the methods of detection using nanoreceptor sensors are compared. Meanwhile, our lab developed electrochemical gustatory biosensors by immobilizing tissues containing the taste buds of rats. The test results for capsaicin and gingerol indicate that the sensitivity and dynamic characteristics exhibited by these two sensors are almost identical. ${ }^{(62)}$ Using these two receptor sensors, we can not only compare the relationship between the artificial signal amplification system and the cell signal amplification system, but also conduct a quantitative study of the interactive dynamics and even the structure-activity relationship between receptors and ligands to achieve the quantitative determination of the gustatory sense using the currently most mature and common electrochemical sensor. ${ }^{(29)}$

In the detection of ORs, because of the research in biochemistry and molecular biology on the transmission of the gustatory signal and its mechanism of action, particularly the need to take G protein-coupled receptors (GPCRs) as the target of drug screening, researchers in medical science have conducted extensive studies on the laws of interaction of ORs and ligands and the laws of their functions in activation/inhibition. Outstanding progress has been made. ${ }^{(63)}$ These techniques are mainly based on the physiological changes in cells caused by the interaction between GPCRs and ligands, for example, the nerve impulse signals generated by the impact of physiological changes on the ion channels (chiefly used for olfactory signal encoding and transmission). ${ }^{(64,65)}$ Unfortunately, these techniques often apply only to a certain specific purpose, and these changes are all dependent on the type and physical activity of cells, the environment of the cells, the interaction between cells, intracellular signaling pathways and their "crosstalk", the control and status of various ion channels, and other complex factors, so it is difficult to realize quantification in a real sense. 
Electrochemical biosensors are different, and the design and detection principle of tissues or cell receptor sensors are shown in Fig. 6. Detecting molecules of different concentrations is like inputting a ligand signal $\mathrm{X}$ into a sensing system. We can detect the output signal $\mathrm{Y}$ of the system and obtain a functional relationship between $\mathrm{Y}$ and $\mathrm{X}$. The signal shows the interaction between receptors and ligands, the interaction of receptors on the cell membrane, the resulting intracellular signal amplification and transmission pathways, and the resulting overall biological effects. Of course, the prerequisite is that there is a clear functional relationship between $\mathrm{X}$ and Y. Obviously, the relationship between $\mathrm{X}$ and $\mathrm{Y}$ is that between an independent variable and a dependent variable, that is, an irreversible causal relationship, which certainly does not exclude positive or negative feedback. Referring to the tissue of the vomeronasal organ of rats, our lab designed a sandwich-structured electrochemical olfactory sensor to detect the gender differences between male and female rats and successfully established an action curve between the concentration of rat urine and the response current. The experimental results indicate that the olfactory sensors of the vomeronasal organs of male and female rats have different dynamic curves for their own urine, and are able to differentiate between their own urine and that of other rats. By adopting liquid chromatography-mass spectrometry (LC-MS) techniques, the main substances causing the smell of the urine of the rats to be different can be further

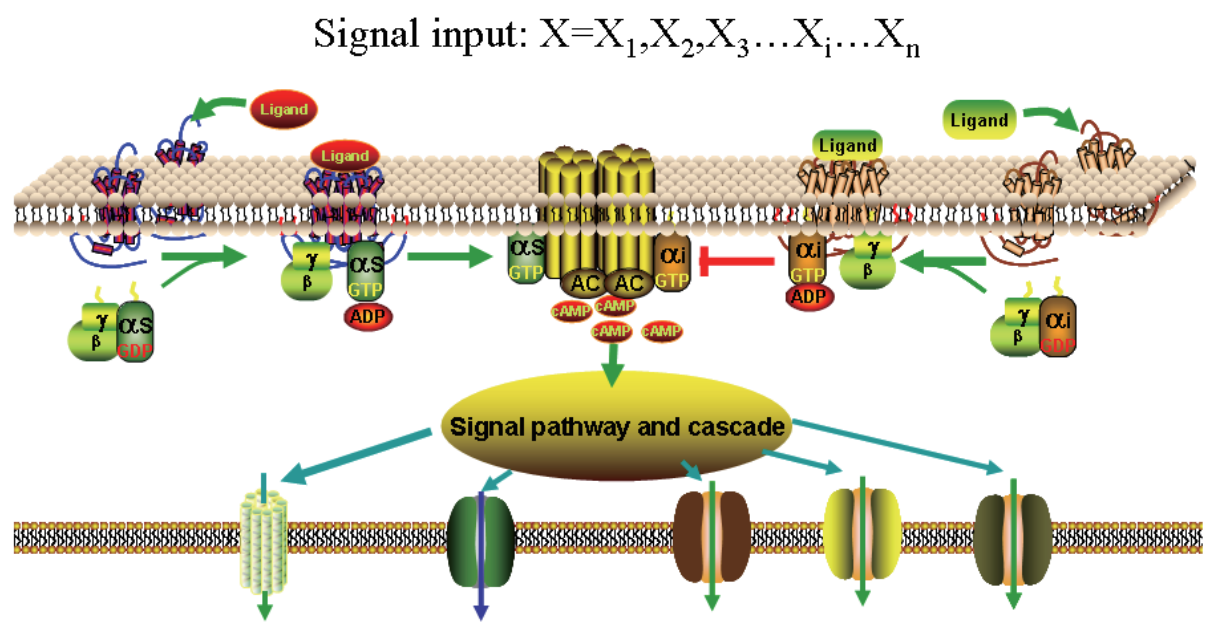

Cytokines, chemokines, hormones and electric signals (ion channels)

Output signal: $\mathrm{Y}=\mathrm{Y}_{1}, \mathrm{Y}_{2}, \mathrm{Y}_{3} \ldots \mathrm{Y}_{\mathrm{i}} \ldots \mathrm{Y}_{\mathrm{n}}$

Fig. 6 (Color online) A schematic diagram of an OR sensor based on cells of a signal amplification system. Biosensors designed using cells and tissues can receive signal input via the receptors on cells and detect output signals through the signal analysis system (including electrochemical-signal-measuring electrodes and immunosensing electrodes). Obviously, by constructing this sensing system and establishing the function of the input variable (an independent variable) $\mathrm{Xi}$ and output variable (a dependent variable) Yi, we can analyze a large amount of information, including the correlation between input signals and output signals, their functional relationship, signal amplification characteristics, the cooperativity among different input signals, and the overall characteristics of signal pathways. 
differentiated, the compounds can be separated from the urine, and the main functions of the compounds can be further determined using electrochemical olfactory sensors and behavioral experiments (unpublished data). The sensor can not only be used in olfactory detection, but also is expected to have very wide application prospects in rodent control and drug screening. Using calcium imaging, Patterson et al. studied the dynamic relationship between odor, concentrationspecific information, and an odor afterimage. The results showed that the dynamics may help identify novel odorants in complex environments. ${ }^{(66)}$

\section{G Protein Signaling Cascade Amplification System}

The OR is the GPCR expressed on the ORNs of the olfactory epithelium, ${ }^{(67)}$ and can bind with odor molecules selectively to activate intracellular signal transduction pathways to cause ORNs to generate action potentials. ${ }^{(68)}$ The most sensitive sensor system is definitely that of insects receiving information from scents in nature, particularly the antenna for sex pheromones. The antennae of insects can sense the semiochemicals released by like creatures within a few hundred meters or even over one thousand meters; our existing instruments cannot realize this level of sensitivity. The antenna sensors of insects depend on the olfaction GPCRs to be activated. When these receptors receive the semiochemicals, they convey the information to the G protein by coupling with GPCRs in the cell. The G protein is the most widespread information amplification device that exists in nature. As a receptor superfamily composed of thousands of members, GPCRs play a crucial role in substance metabolism, energy metabolism, and signal exchange between the body and cells through complex intracellular signaling pathways, so they have always been the focus of attention of scientists. ${ }^{(69)}$ Moreover, GPCRs also carry out "crosstalk" with a number of cellular signaling pathways in performing its important functions in immunoregulation, cell chemotaxis, autophagy, and the control of sexual reproduction, for example. Basically, the seeing, hearing, smelling, and tasting abilities of higher animals, including humans, depend on the transmission function of GPCRs. They can interact with small molecules, such as nutrient, hormone, neurotransmitter, light, and odor molecules, ${ }^{(70)}$ and they are closely related to the activities of human life, making them the key target for filtering therapeutic drugs against diseases in terms of metabolic and nervous systems. ${ }^{(63,71)}$ In the field of medicine, about $25-50 \%$ medicines approved for clinical use become effective by regulating signal transduction of GPCR. ${ }^{(72)}$

According to studies, all GPCRs have the same seven-transmembrane domain, extracellular domain, and intracellular domain. Being very conservative, the intracellular domain is mainly used to activate the $G$ protein signal transmission and amplification system, which constitutes its functional basis. ${ }^{(73)}$ Comparative studies of their extracellular domain prove that they have great diversity, which is a typical example of Darwin's positive choice. This diversity leads to the different ways by which various species obtain nutrition and forms the basis of their survival and their adaptability to different ecological niches. ${ }^{(74,75)}$

The interaction between GPCRs and ligands is very complex and diversified. For example, in the research on the gustatory sensing of GPCRs, the relationship between T1R1 and T1R3 and umami sensing proves that monosodium glutamate (MSG), inosine monophosphate 
(IMP), guanosine monophosphate (GMP) and other umami substances are transmitted by the heterodimer T1R1+T1R3. ${ }^{(76-78)}$ In addition, studies on human and murine transmission of different amino acid, and on their transient calcium responses and normalized chorda tympani responses when amino acids go through taste bud cells show that distinct differences exist in the transmission of "umami" between murines and humans. While murines can transmit the "umami" of almost all amino acids, humans can only transmit the nerve signals of the "umami" of glutamic acid and l-aspartic acid sodium salt (Ajinomoto). ${ }^{(79)}$ In addition, Nelson et al. ${ }^{(76)}$ proved that after the gene of murines is replaced by the human gene for T1R1+3, murines exhibit the same "umami" transmission spectrum as humans. By expressing the $\kappa$-opioid receptor in the taste bud cells of "umami", Zhao et al. ${ }^{(77)}$ further proved that this cell obtained the cell signal ability to transmit opioid substances.

The research on ORs is important in understanding the molecular recognition mechanism of insect chemical signals. Since the first pheromone was identified in the silkworm Bombyx mori, ${ }^{(80)}$ many studies have focused on insect chemical ecology. After several decades of exploration, researchers discovered that in different species, multiple pathways exist for the transduction of ORs signals, and these pathways are regulated and controlled by various ions, amino acids, and small proteins. In vertebrates, the transduction pathway of the cyclic nucleotide cyclic adenosine monophosphate (cAMP), which functions as a second messenger, is a major pathway in the transduction of many odor signals. To date, this pathway has been studied the most thoroughly. The mechanism of this pathway is that the combination of ORs and odor molecules activates the protein Golf inside the cilia, which in turn activates AC3 and catalyzes the counterion of adenosine triphosphate (ATP) in cells to cAMP. ${ }^{(81)}$ cAMP opens the cyclic nucleotide-gated $(\mathrm{CNG})$ ion channel on the cell membrane to guide the inflow of cations (mainly $\mathrm{Ca}^{2+}$ ), and then $\mathrm{Ca}^{2+}$ opens the $\mathrm{Cl}^{-}$pathway, causing the outflow of a large number of $\mathrm{Cl}^{-}$ions to produce action potentials. In addition to the signaling pathways regulated by cAMP, there are other pathways, such as the transduction pathway of inositol trisphosphate, that function as the second messenger. ${ }^{(82)}$ Further research on GPCRs revealed that the olfactory transmission depends mainly on the extracellular domain of receptors; inside the cells, the ways to activate $G$ protein signal amplification and the transduction system, as well as the transduction of ion channels and nerve signals, are the same. In other words, olfactory sensing is encoded by the interaction between receptors and ligands and their distribution and expression on the gustatory tissue. ${ }^{(83)}$ Figure 7 shows the signal transduction process generated from the interaction between ORs and ligands (taken from Ref. 84). (a) In insects, ORN dendrites are encapsulated in sensilla within sensory hairs. Odorant molecules (bound to odorant-binding proteins) access the dendritic surfaces through pores in sensilla. ORN axons in the tissue of insect antennae converge through glomeruli and transduce neural signals to the antennal lobe. (b) ORs in the ORN cell membranes are thought to contain an extracellular $\mathrm{C}$-terminal region, intracellular $\mathrm{N}$-terminal region, and 7 transmembrane domains. Insect ORs have been shown to signal with the aid of chaperone receptors that also regulate the level of OR in the membrane. G-protein-independent ion-channel activity of the chaperone protein (or the OR:chaperone complex) and G-protein mediate increases in intracellular cAMP. IP3 pathways in insects are primarily established in the lobster, although they have been suggested for insects as well. 

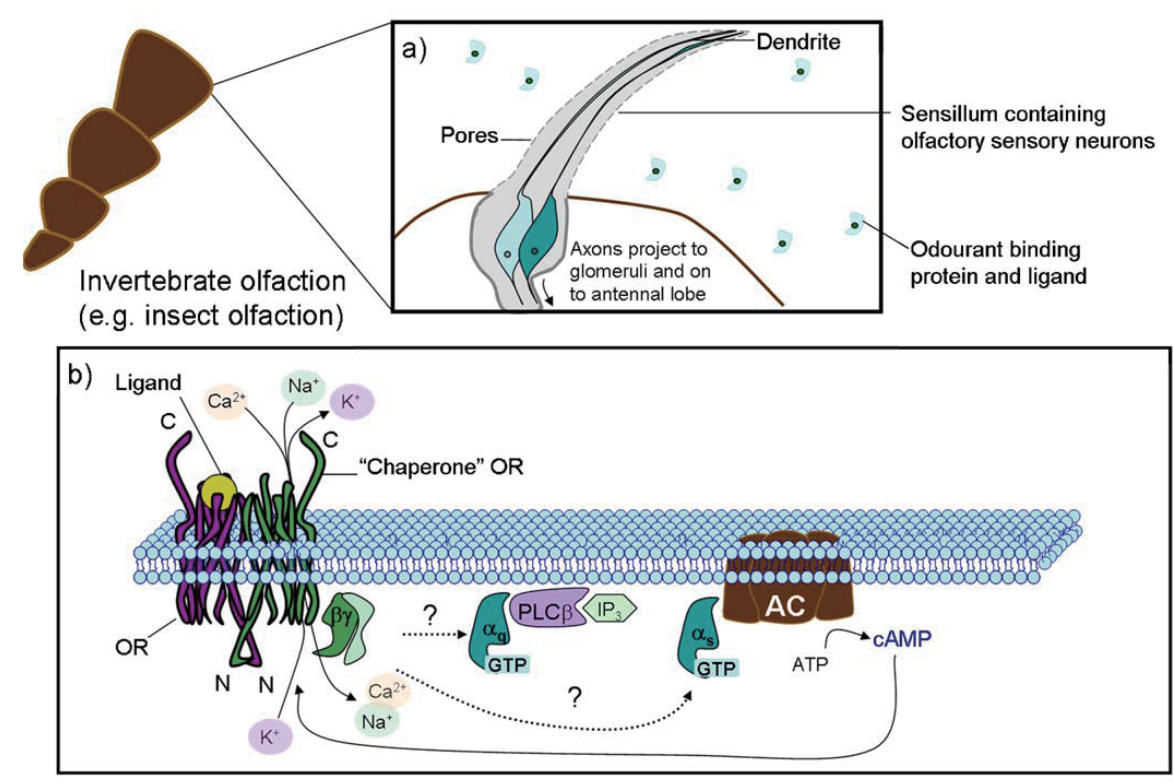

Olfactory receptor neuron dendrite membrane surface

Fig. 7 (Color online) Signal transduction process generated from the interaction between ORs and ligands in insect olfaction. Abbreviations: ORN, olfactory receptor neuron; OR, olfactory receptor; cAMP, cyclic adenosine monophosphate; ATP, adenosine triphosphate; GTP, guanosine triphosphate; AC, adenylyl cyclase III; PLCb, phospholipase c beta; IP3, inositol triphosphate; $\mathrm{iCa}^{2+}$, intracellular calcium.

Wicher et al. ${ }^{(81)}$ showed that odorant stimulation triggers a cyclic nucleotide second messenger system, but Sato et al., ${ }^{(85)}$ undertaking similar experiments, came to the opposite conclusion. Sato et al. did observe cyclic nucleotide sensitivity in some OR-Or83b complexes, but they were unable to demonstrate odorant dependence despite performing similar experiments to those of Wicher et al. Obviously, more work is necessary to elucidate the potential role of second messengers in this system. Both groups do agree, however, in the major finding that ORs are heteromeric ligand-gated cation channels, implying a distinct olfactory signal transduction strategy in insects. It will now be important to establish that this mechanism contributes to odor transduction in insects in vivo.

Under normal circumstances, an extracellular signal-activated receptor can activate multiple $G$ proteins, which redoubles the signals transmitted inside cells; the time the extracellular signals bind with the receptors is relatively short, so that it cannot produce sufficient amplification, while the time of G protein binding with guanosine triphosphate (GTP) and activation is relatively long, about $10-15 \mathrm{~s}$, so after the separation of the receptors and the ligands, G protein can still activate relevant enzyme systems; through the activation of the activity of enzymes in the cascade system and the activity of nonenzymatic proteins, the second messenger controls the life and activities of cells. One amplification is generated for each activation of enzymes or linker molecules. The intracellular signal transduction depends on different series of cascade reactions. ${ }^{(86,87)}$ The amount of GPCR expressed on single cells is very low, and the interaction generated from the binding of receptors and ligands is very weak, but the weak binding signal can be captured eventually by the electrodes because the binding 
of receptors and ligands activates the amplification process of the intracellular $G$ protein, causing a series of changes in electrochemical signals. This realized the identification and detection of gas molecules and also confirmed that the $G$ protein signal amplification system could be used to sense the signals transmitted by the GPCRs of other species. Jin et al. ${ }^{(88)}$ reported that the immobilized expression on the surface by SPR included b2-adrenoceptor of human embryoic kidney-293 cells, indicating that the detection of the ligand isoprenaline was realized. By adding the inhibitor LY29402 of phosphatidylinositol 3-hydroxy kinase (PI3K) (the second messenger during the signaling process of a $G$ protein), the sensitivity of the sensor to isoprenaline tripled. This further illustrates that the application of a $G$ protein signaling cascade in biosensors has very broad prospects, as has been reported by researchers. ${ }^{\left({ }^{89}\right)}$ On the basis of tissue from the taste buds of pigs, Wei et al. in our lab designed a sandwich-structured electrochemical sensor to test bitterness, and the test results indicated that the signal can be amplified over ten thousand times (unpublished).

We believe that the use of the $G$ protein amplification system will play an important role in aspects such as quantitative issues of receptor-ligand interactions, the development of taste and olfactory sensors, and drug screening.

\section{Quantitative Problems of Testing Olfactory Samples}

Currently, the commonly used pretreatment method for olfactory samples is to use specialized air collection devices or to allow the samples to volatilize by dropping them onto filter paper for testing. Being greatly influenced by external environmental factors, including gas flow, temperature, and the inherent defects of devices, the quantification of the samples has been very difficult. Scott et al. ${ }^{(90)}$ studied the solubility and sorption pattern in olfactory epithelial responses and found that the retronasal and orthonasal electro-olfactogram (EOG) response is activated by an odor that mainly dissolves in the mucosal fluids and then is adsorbed by the olfactory epithelium. Odor information is first represented as a spatial activation pattern across the olfactory epithelium, when an odor is drawn into the nose by breathing. This epithelial pattern likely results from both the intrinsic OSN sensitivity and the sorptive patterns imposed by the interaction of nasal aerodynamics with physiochemical properties of odorants, although the precise contributions of each are ill-defined. Misawa et al. ${ }^{(91)}$ reported a highly sensitive and selective chemical sensor using living cells (Xenopus laevis oocytes) within a portable fluidic device. This odorant sensor's sensitivity was a few parts per billion in solution and the sensor could simultaneously distinguish different types of chemicals that have only slight differences in double-bond isomerism or functional groups such as $-\mathrm{OH}$, $-\mathrm{CHO}$, and $-\mathrm{C}(=\mathrm{O})-$. In addition, they found that the sensor was effective for multiple-target chemicals and can be integrated with a robotic system without the need for any noise reduction systems. This means that, before testing, some olfactory substances can be dissolved in water or organic solvents to clarify the standardized concentration of the samples and to solve the quantitative problems of odorant detection. 


\section{Summary and Outlook}

We have reviewed the most recent progress in OR-based biosensors, emphasizing critical issues related to their development and transduction techniques. Because of their significant advantages of high sensitivity and specificity, OR-based biosensors hold great potential to be developed into bioelectronic nose systems for the detection of VOCs in many fields, including food safety, environmental and industrial monitoring, clinical diagnosis, agricultural disease, and drug discovery. Thus far, more than $40 \%$ of drugs used clinically are the results of GPCR screening. However, because the structures and functions of GPCRs of different species tend to be similar and conserved, it is difficult for the drugs screened using GPCRs (such as cancer drugs and antiparasitics) to have selective killing effects, which severely restricts the development and screening of new drugs. ${ }^{(63)}$ Studies have revealed that a large difference exists among the functions of the olfactory GPCRs of different species, for example, the identification function for sex pheromones of different insects is significantly different. Therefore, utilizing the functional differences of olfactory GPCRs in drug screening should have very broad prospects. ${ }^{(92)}$ Because of the important roles olfactory substances play in nature, the research and development of olfactory sensors using OR cells or tissues as the recognition element is receiving the attention of more and more researchers. ${ }^{(27)}$ In a number of olfactory sensors developed on the basis of the changes in light, electricity, heat, and sound ${ }^{(84)}$ caused by interactions between the receptors and ligands, the electrochemical sensors of ORs can take full advantage of the signaling cascade amplification system of the cells themselves. By combining electrochemical signal amplification methods using enzymes and nanomaterials, OR electrochemical sensors enable us directly study interactions between receptors and ligands of living cells, tissues, and organs, as well as the laws governing the output of the signals of cell activity through the intracellular signaling cascade amplification system. This has great significance in the screening of GPCR drugs. In addition, the OR sensor has extensive application prospects in insect and rodent control (using sex pheromones), and also has considerable potential for applications in public safety and environmental monitoring. Moreover, OR-based biosensors also provide a useful platform for basic olfactory research by enabling the detection of specific interactions between ORs and odorants, such as receptor deorphanization, antagonism, and olfactory signal decoding.

\section{Acknowledgments}

This work was supported by the National Natural Science Foundation of China (Nos. 31671857 and 31371773).

\section{References}

1 J. H. Fabre: Souvenirs entomologiques: e'tude sur l'instinct et les moeurs des insectes, Vol. 2 (Robert Laffont, Paris, 1989) p. 1.

2 P. Rau and N. Rau: Trans. Acad. Sci. St. Louis 26 (1929) 83.

3 A. Sampat, M. Lopatka, M. Sjerps, G. Vivo-Truyols, P. Schoenmakers, and A. Asten: TrAC-Trend Anal. Chem. 80 (2016) 345. 
4 G. A. Eiceman, J. Gardea-Torresday, E. Overton, K. Carney, and F. Dorman: Anal. Chem. 78 (2006) 3985.

5 Y. Sapozhnikova and S. J. Lehotay: Anal. Chim. Acta 899 (2015) 13.

6 S. Zaromb and J. R. Stetter: Sens. Actuators 6 (1984) 225.

7 K. Persaud and G. Dodd: Nature 299 (1982) 352.

8 J. W. Gardner and P. N. Bartlett: Electronic Noses: Principles and Application (Oxford University Press, New York, 1999) p. 2.

9 K. Pradeep: An Electronic Nose for Detecting Hazardous Chemicals and Explosives, Plant Growth Modeling, Simulation, Visualization and Applications (PMA) (International Symposium, Waltham, MA, USA, 2006).

10 Supriyadi, K. Shimizu, M. Suzuki, K. Yoshida, T. Muto, A. Fujita, N. Tomita, and N. Watanabe: Flavour Fragance J. 19 (2004) 44.

11 S. Buratti, D. Ballabio, S. Benedetti, and M. S. Cosio: Food Chem. 100 (2007) 211.

12 M. Tanaka, H. Anguri, A. Nonaka, K. Kataoka, H. Nagata, J. Kita, and S. Shizukuishi: J. Dent. Res. 83 (2004) 317.

13 J. B. Yu, H. G. Byun, M. S. So, and J. S. Huh: Sens. Actuators, B 108 (2005) 305.

14 E. I. Mohamed, R. Linder, G. Perriello, N. Di-Daniele, S. J. Pöppl, and A. De-Lorenzo: Diabetes Nutr. Metab. 15 (2002) 215.

15 M. E. Shykhon, D. W. Morgan, R. Dutta, E. L. Hines, and J. W. Gardner: Am. J. Otolaryngol. 118 (2004) 706.

16 S. Ohmori, Y. Ohno, T. Makino, and T. Kashihara: Eur. J. Pharm. Biopharm. 59 (2005) 289.

17 A. Voss, V. Baier, R. Reisch, K. Von-Roda, P. Elsner, H. Ahlers, and G. Stein: Ann. Biomed. Eng. 33 (2005) 656.

18 C. Di Natale, R. Paolesse, G. D’Arcangelo, P. Comandini, G. Pennazza, E. Martinelli, S. Rullo, M. C. Roscioni, C. Roscioni, A. Finazzi-Agrò, and A. D’Amico: Med. Sci. Monit. 11 (2005) 366.

19 M. Bernabei, G. Pennazza, M. Santonico, and A. D’Amico: Sens. Actuators, B 131 (2008) 1.

20 A. D’Amico, R. Bono, G. Pennazza, M. Santonico, G. Mantini, M. Bernabei, M. Zarlenga, C. Roscioni, E. Martinelli, R. Paolesse, and C. Di-Natale: Skin Res. Technol. 14 (2008) 226.

21 R. F. Machado, D. Laskowski, O. Deffenderfer, T. Burch, S. Zheng, P. J. Mazzone, T. Mekhail, C. Jennings, J. K. Stoller, J. Pyle, J. Duncan, R. A. Dweik, and S. C. Erzurum: Am. J. Respir. Crit. Care Med. 171 (2005) 1286.

22 Y. Shen, B. Zhou, and S. Wang: Acta Optica Sinica 28 (2008) 1934.

23 X. Gao, W. Huang, and Z. Li: Acta Optica Sinica 23 (2003) 609.

24 R. Kan, W. Liu, and Y. Zhang: Acta Optica Sinica 26 (2006) 67.

25 J. Chen, J. Liu, and Y. Zhang: Acta Optica Sinica 27 (2007) 350.

26 Y. Liu, X. Chen, and A. Ouyang: Acta Optica Sinica 28 (2008) 478.

27 L. Du, C. Wu, Q. Liu, L. Huang, and P. Wang: Biosens. Bioelectron. 42 (2013) 570.

28 H. Y. Zhang, H. Q. Pan, B. L. Zhang, and J. L. Tang: Chin. J. Anal. Chem. 40 (2012) 801.

29 X. Y. Wang and G. C. Pang: Sens. Rev. 35 (2015) 30.

30 S. Singamaneni, M. C. Lemienux, H. P. Lang, C. Gerber, Y. Lam, S. Zauscher, P. G. Datskosm, N. V. Lavrik, H. Jiang, and R. R. Naik: Adv. Mater. 20 (2008) 653.

31 P. M. Kosaka, V. Pini, J. J. Ruz, R. A. Da-Silva, M. U. Gonzalez, D. Ramos, M. Calleja, and J. Tamayo: Nat. Nanotechnol. 9 (2015) 1047.

32 B. Johnson and R. Mutharasan: Analyst 139 (2014) 1112.

33 K. Neethu and K. J. Suja: Procedia Comput. Sci. 93 (2016) 146.

34 Z. Tan, N. Zhang, and J. Li: Acta Mech. Solida Sin. 27 (2014) 122.

35 M. Andrew and B. Thomas: Bioinspir. Biomim. 6 (2011) 016002.

36 X. Wang, J. Xu, Y. L. Shen, F. Y. Liu, and Y. J. Du: Chin. J. Appl. Ecol. 20 (2009) 1973.

37 E. Ngumbi, L. Chen, and H. Y. Fadamiro: J. Chem. Ecol. 35 (2009) 1009.

38 D. Schneider: Z. Vgl. Physiol. 40 (1957) 8.

39 T. Røstelien, A. K. Borg-Karlson, J. Fäldt, U. Jacobsson, and H. Mustaparta: Chem. Senses 25 (1999) 141.

40 N. J. Vickers and T. Baker: Proc. Natl. Acad. Sci. U.S.A. 91 (1994) 5756.

41 J. Bau, K. A. Justus, and R. T. Cardé: J. Insect Physiol. 48 (2002) 433.

42 N. J. Vickers, T. A. Christensen, T. C. Baker, and J. G. Hildebrand: Nature 410 (2001) 466.

43 D. M. Light, E. B. Jang, and R. A. Flath: Entomol. Exp. Appl. 63 (1992) 13.

44 P. Skládal: TrAC, Trends Anal. Chem. 79 (2016) 127.

45 A. Sun, Y. Yang, Y. L. Jiang, Z. Fan, Q. Liu, and Q. Zhou: Sens. Actuators, B 66 (2000) 88.

46 L. Du, C. Wu, H. Peng, L. Zou, L. Zhao, L. Huang, and P. Wang: Sens. Actuators, B 187 (2013) 481.

47 P. Fromherz, A. Offenhäusser, T. Vetter, and J. Weis: Science 252 (1991) 1290.

48 S. Schütz, M. J. Schoning, P. Schroth, Ü. Malkoc, V. Weißbecker, P. Kordos, H. Lüth, and H. E. Hummel: Sens. Actuators, B 65 (2000) 291. 
M. J. Huotari: Sens. Actuators, B 71 (2000) 212.

50 K. Shimizu and M. Stopfer: Nature 492 (2012) 44.

51 A. B. Ismail, T. Yoshinobu, H. Iwasaki, H. Sugihara, T. Yukimasa, I. Hirata, and H. Iwata: Biosens. Bioelectron. 18 (2003) 1509.

52 B. Stein, M. George, H. E. Gaub, and W. J. Parak: Sens. Actuators, B 98 (2004) 299.

53 G. Xu, X. Ye, L. Qin, Y. Xu, Y. Li, R. Li, and P. Wang: Biosens. Bioelectron. 20 (2005) 1757.

54 Q. Liu, H. Cai, Y. Xu, Y. Li, R. Li, and P. Wang: Biosens. Bioelectron. 22 (2006) 318.

55 J. A. Ribeiro, P. M. Fernandes, C. M. Pereira, and F. Silva: Talanta 160 (2016) 653.

56 L. Reverté, B. Prieto-Simón, and B. Campàs: Anal. Chim. Acta 908 (2016) 8.

57 J. Anzai: Mater. Sci. Eng., C 67 (2016) 737.

58 S. Shrivastava, N. Jadon, and R. Jain: TrAC, Trends Anal. Chem. 82 (2016) 55.

59 X. B. Kang, G. C. Pang, X. Y. Liang, M. Wang, J. Liu, and W. M. Zhu: Electrochim. Acta 62 (2012) 327.

60 D. Q. Lu, F. P. Lu, and G. C. Pang: Biomed. Microdevices 18 (2016) 50.

61 X. B. Kang, G. C. Pang, Q. S. Chen, and X. Y. Liang: Sens. Actuators, B 177 (2013) 1010.

62 L. Qiao, L. Jiao, G. C. Pang, and J. B. Xie: Biosens. Bioelectron. 68 (2015) 454.

63 B. Shoichet and B. Kobilka: Trends Pharmacol. Sci. 33 (2012) 268.

64 M. R. Whorton and R. MacKinnon: Nature 498 (2013) 190.

65 C. Y. Su, K. Menuz, J. Reisert, and J. R. Carlson: Nature 492 (2012) 66.

66 M. A. Patterson, S. Lagier, and A. Carleton: PNAS 110 (2013) 3340.

67 M. Spehr and S. D. Munger: J. Neurochem. 109 (2009) 1570.

68 I. Gaillard, S. Rouquier, and D. Giorgi: Cell Mol. Life Sci. 61 (2004) 456.

69 R. Fredriksson, M. C. Lagerström, L. G. Lundin, and H. B. Schiöth: Mol. Pharmacol. 63 (2003) 1256.

70 D. M. Rosenbaum, S. G. Rasmussen, and B. K. Kobilka: Nature 459 (2009) 7245.

71 B. C. Heng, D. Aubel, and M. Fussenegger: Biotechnol. Adv. 31 (2013) 1676.

72 A. L. Parrill and D. L. Bautista: Pharmaceuticals 4 (2010) 7.

73 M. Audet and M. Bouvier: Cell 151 (2012) 14.

74 K. M. Schlinkmanna, A. Honegger, E. Türeci, K. E. Robison, D. Lipovšek, and A. Plückthun: PNAS 109 (2012) 9810.

75 I. Dodevski and A. Plückthun: J. Mol. Biol. 408 (2011) 599.

76 G. Nelson, J. Chandrashekar, M. A. Hoon, L. Feng, G. Zhao, N. Ryba, and C. S. Zuker: Nature 416 (2002) 199.

77 G. Q. Zhao, Y. Zhang, M. A. Hoon, J. Chandrashekar, I. Erlenbach, N. Ryba, and C. S. Zuker: Cell 115 (2003) 255.

78 X. Li, L. Staszewski, H. Xu, K. Durick, M. Zoller, and E. Adler: PNAS 99 (2002) 4692.

79 J. A. Maga: Crit. Rev. Food Sci. Nutr. 18 (1983) 231.

80 A. Butenandt, U. Groschel, P. Karslon, and W. Zillig: Arch. Biochem. Biophys. 83 (1959) 76.

81 D. Wicher, R. Schafer, R. Bauernfeind, M. C. Stensmyr, R. Heller, S. H. Heinemann, and B. S. Hansson Nature 452 (2008) 1007.

82 T. Shiraiwa, M. Kashiwayanagi, T. Lijima, and M. Murakami: Biochem. Biophys. Res. Commun. 355 (2007) 1019.

83 W. Bradford, A. Buckholz, J. Morton, C. Price, A. M. Jones, and D. Urano: Sci. Signal. 6 (2013) 1.

84 R. Glatz and K. Bailey-Hill: Prog. Neurobiol. 93 (2011) 270.

85 K. Sato, M. Pellegrino, T. Nakagawa, L. B. Vosshall, and K. Touhara: Nature 452 (2008) 1002.

86 S. R. Neves, P. T. Ram, and R. Iyengar: Science 296 (2002) 1636.

87 Y. Gu, P. Lucas, and J. P. Rospars: PLoS Comput. Biol. 5 (2009) e1000321.

88 B. Jin, X. Ji, and N. Toshio: Electrochim. Acta 50 (2004) 1049.

89 W. R. Leifert, R. V. Glatz, K. Bailey, T. Cooper, M. Bally, B. M. Stadler, E. Reimhult, and J. G. Shapter: Annual Review of Nano Research: Nanoscale Biosensors and Biochips (World Scientific Publishing Co. Pte. Ltd., Singapore, 2009) p. 1.

90 J. W. Scott, L. Sherrill, J. Jiang, and K. Zhao: J. Neurosci. 34 (2014) 2025.

91 N. Misawa, H. Mitsuno, R. Kanzaki, and S. Takeuchi: PNAS 107 (2010) 15340.

92 S. R. Foster, E. Roura, and W. G. Thomas: Pharmacol. Ther. 142 (2014) 41. 